\title{
Filigrane
}

Écoutes psychothérapiques

\section{Un rêve-manifeste}

\section{Nicolas Lévesque}

Volume 20, numéro 1, printemps 2011

URI : https://id.erudit.org/iderudit/1004041ar

DOI : https://doi.org/10.7202/1004041ar

Aller au sommaire du numéro

\section{Éditeur(s)}

Revue Santé mentale au Québec

ISSN

1192-1412 (imprimé)

1911-4656 (numérique)

Découvrir la revue

Citer cet article

Lévesque, N. (2011). Un rêve-manifeste. Filigrane, 20(1), 77-79.

https://doi.org/10.7202/1004041ar d'utilisation que vous pouvez consulter en ligne.

https://apropos.erudit.org/fr/usagers/politique-dutilisation/ 


\title{
Un rêve-manifeste
}

\author{
Nicolas Lévesque
}

C'est arrivé un soir après une journée chargée, à la suite d'un nombre de séances suffisant pour qu'advienne cet état où je ne m'appartenais plus, dégagé de moi-même, en exil dans tous ces psychismes, totalement ouvert à la nuit, aux étoiles, à l'odeur de bois brûlé que l'on sent parfois, certains soirs d'hiver. Je marchais avec ce second souffle, très étrange, qui succède à un premier plateau de fatigue. Voilà : ma pratique avait atteint une maturité. Je voyais que mes patients le sentaient, que j'étais réellement devenu un psychanalyste. Mais comment cela se pouvait-il? Je projetais pourtant d'attendre d'avoir 50 ans, plus de temps, plus d'argent, des enfants devenus grands, avant de m'engager, de m'inscrire, de souscrire, à un Institut ou une École, comme on dit. Pour la première fois, je ne remettais plus en doute mon statut de psychanalyste, tout en ne me permettant pas d'écrire ce titre sous mon nom, sur la porte de mon bureau de psychologue. Ce qui me préoccupait, ce soir-là, c'était la possibilité de devenir un «vrai» psychanalyste sans être passé par une institution analytique. J'incarnais, ce soir-là, cette possibilité. Et cela me troublait. Quelque chose de la présence de la psychanalyse à l'Université avait réussi à se frayer un chemin en moi, malgré tous les vents défavorables. J'étais toutefois né, en tant que psychanalyste, dans un drôle de contexte, une étrange scène primitive qui produit des psychanalystes-qui-n'en-sont-pas et des Écoles, des Instituts, vidés de toute relève réelle. La peste avait fait son cuvre; j'étais isolé, en quarantaine, être hybride, bâtard, tabou: psychanalyste, à 36 ans, sans affiliation et libre d'association. Mais ce qui me troublait le plus, c'était le visage de tous ces jeunes que j'ai connu (étudiant(e) s, patient(e) s) en qui naissaient une authentique "vocation" analytique et à qui je n'ai pu donner que quelques numéros de téléphone, tout en sachant très bien ce qui les attendait, le constat cruel de véritables passions psychanalytiques tuées dans l'œuf... Et j'ai fait la nuit dernière ce rêve que je ne peux plus garder pour moi seul; le contenu manifeste du rêve contenait ce manifeste.

\section{Manifeste pour un département de psychanalyse au Québec}

es instituts psychanalytiques se vident de toute jeunesse et l'Université produit des psychiatres et psychologues «d'approche psycho-dynamique». Dans quel état laisserons-nous la psychanalyse aux prochaines générations? Comment installer un cadre pour la transmission de la psychanalyse? Voici une idée qui semble plus pertinente que jamais : la création d'un département de psychanalyse au sein d'une université québécoise.

Cela ouvrirait la possibilité de commencer sa formation beaucoup plus jeune. Car dans la structure actuelle, au moment où, pour plusieurs, à la fin d'un parcours universitaire, vient l'envie de fonder une famille, le besoin de se trouver un emploi, 
celui aussi de faire enfin l'expérience d'une liberté et d'une indépendance professionnelle longtemps attendue, est-il vraiment sain de proposer, à cette étape de la vie, une autre formation institutionnelle et un autre fardeau financier (pour qui n'est pas médecin)? Bien sûr que non.

Avec un département universitaire, l'on pourrait construire l'indépendance de la psychanalyse par rapport à la psychiatrie et la psychologie, notamment. Pour ce faire, il faudrait un programme qui comporte à la fois une dimension de recherche fondamentale (thèses de doctorat, par exemple) et un volet professionnel (M. Ps. ? D. Ps. ?), qui donnerait accès à la profession clinique de psychanalyste. Oui, la «profession de psychanalyste» (et pourquoi pas encadrée par quelque chose qui ressemblerait à l'Ordre des psychologues ou au Collège des médecins, mais qui respecterait la singularité psychanalytique). Oui, une identité officielle, non plus un second titre ou un titre à cacher sous un autre ou le maquillage honteux du «psychodynamique». Ce serait une reconnaissance symbolique, le symbole d'une discipline qui, après plus de cent ans, mérite cette intégration à une société à laquelle elle a beaucoup à donner. Une psychanalyse qui n'aurait plus à chercher refuge un peut partout, qui aurait enfin un chez soi, un droit de cité officiel. Cet accès à une profession (autonome, en dehors des nouveaux paramètres du «psychothérapeute »), à un emploi rémunéré, est le nerf de la guerre, le levier de liberté et d'indépendance nécessaire. La psychanalyse vit actuellement un compromis névrotique trop insatisfaisant, un statu quo paralysant, subissant tous les aspects négatifs de l'institutionnalisation, sans profiter des aspects positifs. Les extrêmes offrent le meilleur des deux mondes: d'une part, un programme universitaire et, d'autre part, des espaces vraiment hors institution pour tout le reste (communautés de pensée, de lecture, d'écriture, d'affinités cliniques, libres de toute affiliation, ce qui ne manquerait pas de favoriser une plus grande créativité clinique et théorique).

Dans le même élan, il s'agirait de libérer l'analyse personnelle de toute intrusion institutionnelle; une loi non écrite stipulerait bien entendu que chaque analyste devrait avoir fait (et continuer de faire) l'expérience de son propre inconscient, mais ce serait sa liberté, son espace hors d'atteinte, hors de contrôle et de portée du «parent» institutionnel. Il s'agirait ici de faire confiance au futur analyste. Les critères habituels (nombre d'années, fréquence des séances, analyste didacticien, etc.) n'ont jamais assuré la garantie de quoi que ce soit.

La clinique de psychanalyse populaire du département permettrait de rétablir le lien avec la population en offrant des analyses à bas tarifs (et au cadre flexible, déterminé par la singularité du cas), tout en assurant un revenu au psychanalyste en formation. Elle serait le lieu privilégié de la transmission intergénérationnelle de l'expérience clinique et la mise à l'épreuve du bagage théorique.

Héritages freudien, lacanien, derridien, kleinien, winnicottien, pour ne nommer que ceux-là, trouveraient refuge ensemble dans un département hétérogène qui permettrait les différences, les différents destins de l'histoire de la psychanalyse et ses rapports avec les autres disciplines. Vivement une solidarité psychanalytique!

Avec un appui suffisant, ce projet - qui n'est encore, à ce stade-ci, qu'une idée, qu'un rêve, et qui pourrait être porté par des gens qui ont plus de talent politique que 
moi - pourrait s'amorcer avec des démarches plus concrètes et se confronter avec ferveur et patience, étape par étape, à l'épreuve de réalité.

Pour des appuis ou des commentaires, vous pouvez m'écrire par courriel.

Je fais d'étranges rêves, il est vrai. Je ne sais même pas si je dois les prendre au sérieux, voire les prolonger dans une action, une réalité, un projet. Mais j'avais besoin de raconter celui-ci aux amis de la psychanalyse. Qui sait? Peut-être certains font-ils le même genre de rêves.

Nicolas Lévesque 960, rue Jean-Talon Est, app. 4

Montréal

H2R 1V4

niclev@total.net 